\title{
Dietary and supplemental antioxidant and anti-inflammatory nutrient intakes and pulmonary function
}

\author{
Tze Pin $\mathrm{Ng}^{1,2, *}$, Mathew Niti ${ }^{1,3}$, Keng Bee Yap ${ }^{1,4}$ and Wan Cheng Tan ${ }^{1,5}$ \\ 'Gerontological Research Programme, Yong Loo Lin School of Medicine, National University of Singapore, \\ Singapore: ${ }^{2}$ Department of Psychological Medicine, National University Hospital, Yong Loo Lin School of \\ Medicine, National University of Singapore, 5 Lower Kent Ridge Road, Singapore 119074: ${ }^{3}$ Health Service \\ Research and Evaluation, Ministry of Health, Singapore: ${ }^{4}$ Alexandra Hospital, Singapore: ${ }^{5}$ Respiratory Medicine \\ Division, University of British Columbia, Vancouver, British Columbia, Canada
}

Submitted 4 January 2013: Final revision received 16 August 2013: Accepted 20 August 2013: First published online 27 September 2013

\begin{abstract}
Objective: A limited but growing body of evidence supports a significant role of antioxidant and anti-inflammatory micronutrients in pulmonary health. We investigated the associations of dietary and supplemental intakes of vitamins A, C, $\mathrm{E}$ and D, Se and $n$-3 PUFA with pulmonary function in a population-based study. Design: Population-based, cross-sectional study and data analysis of fruits and vegetables, dairy products and fish, vitamins $\mathrm{A}, \mathrm{C}, \mathrm{E}$ and $\mathrm{D}$, Se and $n-3$ PUFA supplemental intakes, pulmonary risk factors and spirometry.

Subjects: Chinese older adults ( $n$ 2478) aged 55 years and above in the Singapore Longitudinal Ageing Studies.

Results: In multiple regression models that controlled simultaneously for gender, age, height, smoking, occupational exposure and history of asthma/chronic obstructive pulmonary disease, BMI, physical activity, and in the presence of other nutrient variables, daily supplementary vitamins A/C/E $(b=0 \cdot 044, \mathrm{sE}=0 \cdot 022$, $P=0 \cdot 04)$, dietary fish intake at least thrice weekly $(b=0 \cdot 058$, sE $=0 \cdot 016$, $P<0 \cdot 0001)$ and daily supplementary $n$-3 PUFA $(b=0 \cdot 068$, sE $=0 \cdot 032, P=0 \cdot 034)$ were individually associated with forced expiratory volume in the first second. Supplemental $n$-3 PUFA was also positively associated with forced vital capacity ( $b=0 \cdot 091, \mathrm{sE}=0 \cdot 045, P=0 \cdot 045)$. No significant association with daily dairy product intake, vitamin D or Se supplements was observed.

Conclusions: The findings support the roles of antioxidant vitamins and $n$ - 3 PUFA in the pulmonary health of older persons.
\end{abstract}

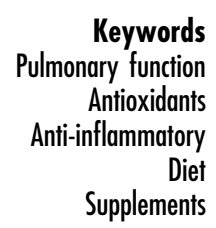

Among the elderly, greater exposure to oxidative stress particularly from smoking and lower dietary intake of antioxidant nutrients in foods such as fresh fruits and vegetables, milk and fish may render them particularly vulnerable to lung damage, thus increasing the risk of chronic obstructive pulmonary disease (COPD) in late life. A limited but growing body of evidence suggests a significant role of antioxidant and anti-inflammatory nutrients in protecting the lungs from the effects of oxidative stress and chronic inflammation ${ }^{(1,2)}$.

Studies based on FFQ measures of dietary intake of fresh fruits and vegetables ${ }^{(3-10)}$, supported by studies with serum measurements of micronutrients such as vitamin A, vitamin C and vitamin $\mathrm{E}$ and other phytochemicals ${ }^{(11-14)}$, suggest that antioxidant vitamins $\mathrm{A}, \mathrm{C}$ and $\mathrm{E}$ are associated with better pulmonary function. It has also been suggested that serum Se, another antioxidant, may have pulmonary protective effects but its reported associations with pulmonary function are based on a limited number of studies ${ }^{(11,15)}$.
Vitamin D (1,25-dihydroxy) inhibits the formation of matrix metalloproteinases as well as fibroblast proliferation, and influences collagen synthesis, and may therefore play a role in influencing pulmonary tissue remodelling ${ }^{(16,17)}$. A recent study reported that serum concentrations of $1,25-$ dihydroxyvitamin D were positively associated with forced expiratory volume in the first second $\left(\mathrm{FEV}_{1}\right)$ and forced vital capacity $(\mathrm{FVC})^{(18)}$, but this finding was not replicated independently in a subsequent study ${ }^{(19)}$.

$n-3$ PUFA are components of cell membranes and play an important role in suppressing the production of pro-inflammatory mediators such as $\mathrm{PGE}_{2}$ and leukotriene $\mathrm{B}_{4}{ }^{(20)}$, and may thus have a protective effect against pulmonary function deterioration. The reported associations between fish intake (a rich source of $n-3$ PUFA) and pulmonary function is equivocal and based on sparse data from a relatively small number of studies ${ }^{(4,5,21-23)}$.

Of note, most previous studies did not control simultaneously for parallel intake of other antioxidant or 
anti-inflammatory nutrients; hence a protective effect attributed to one antioxidant or micronutrient may actually reflect the effect of another correlated dietary constituent or an interaction between dietary constituents. In the present study, we investigated the separate and joint effects of high dietary intakes of fruits and vegetables, milk and dairy products, and fish, and supplemental intakes of vitamins A, C, E and D, Se and $n$-3 PUFA, with pulmonary function in a large population sample of Chinese older adults in Singapore.

\section{Methods}

\section{Study participants}

The study sample was drawn from participants in the Singapore Longitudinal Ageing Studies (SLAS), an observational cohort study of ageing and health among community-dwelling elderly persons. From September 2003 to December 2004, participants aged 55 years and above were recruited by door-to-door census ( $n$ 3894) from the whole population residing in five districts in the South East Region of Singapore, excluding those who were severely incapacitated physically or mentally and unable to give informed consent or participate. A total of 2804 residents participated in the study (estimated response rate $78 \%$ ). The study was approved by the National University of Singapore Institutional Review Board. Consenting participants underwent extensive interviews and examinations that included measurements of lung function. Among the 2608 Chinese respondents in the cohort, eighty-one respondents who did not perform spirometry, forty-six with technically unsatisfactory spirometric performance and three with missing data were excluded from the analysis. Complete spirometric data were analysed for 2478 respondents.

\section{Spirometry}

Ventilatory function testing was performed using a portable, battery operated, ultrasound transit-time based spirometer (Easy-One model 2001 Diagnostic Spirometer; NDD Medical Technologies, Zurich, Switzerland). Forced expiratory manoeuvres were performed with the respondent seated according to the American Thoracic Society recommendations on standardization of procedures: at least three technically acceptable manoeuvres, with the two best FVC and $\mathrm{FEV}_{1}$ readings reproducible to within 5\% or $200 \mathrm{ml}$. The largest $\mathrm{FEV}_{1}$ and the largest FVC on any of the acceptable tests were used. Height and weight were measured with a portable Seca stadiometer (model 708 1314004; Vogel \& Hake, Hamburg, Germany).

\section{Questionnaires}

A semi-quantitative FFQ was used to ask participants whether over a 1-month period they drank or ate 'a lot of' milk products (at least one serving every day); 'a lot of' fruits or vegetables (at least one serving every day); and 'a lot of' fish (more than three times per week).

For supplemental intake, participants were asked the frequencies with which they regularly consumed vitamins $\mathrm{A}, \mathrm{C}, \mathrm{E}$ or $\mathrm{D}$, Se and $n-3$ PUFA (DHA, EPA) supplements: (i) 'never or rarely'; (ii) 'less than once a month'; (iii) 'more than once a month but less than one time a week'; (iv) 'more than once a week but not daily'; or (v) 'always (daily)'. The distributions were extremely bimodal; the vast majority were for (i) 'never or rarely' or (v) 'daily' and fewer than $6 \%$ of the responses were for intermediate frequencies. Hence, the responses were dichotomized by daily intake of supplements (yes/no).

Other data included risk factors known to determine ventilatory function and the risk of COPD, namely age, gender, housing type (an established surrogate measure of socio-economic and income status), smoking (never, past or current smoker), past occupational exposure to dust or fumes, and reported medical history of asthma or COPD diagnosis. The usual frequency of three categories of moderate-to-vigorous physical activity (including walking, physical exercises and sports activities) reported was scored as $1=$ 'never or rarely (less often than once a month)', 2 = 'sometimes (at least once a month but less than once week)' and $3=$ 'often (once a week or more)', and the summed scores were categorized into four levels from 1 (score $=0$, equivalent to never or rarely for any physical activity) to 4 (score $=6$ or more, equivalent to at least once weekly for two or more activities).

\section{Statistical analysis}

Multiple linear regression techniques were used to investigate the association between dietary and supplemental intakes (as independent categorical variables) and $\mathrm{FEV}_{1}$, $\mathrm{FVC}$ or $\mathrm{FEV}_{1} / \mathrm{FVC}$ (as dependent continuous variables). Gender, age (single years), height $(\mathrm{cm})$, smoking status (non-smokers, past smoker, current smoker), past occupational history and reported medical history of asthma and/or COPD, BMI and the level of physical activity were included as variables in the base model. The individual associations of each dietary and supplemental intake variable with ventilatory function were investigated separately (model 1) in the presence of variables in the base model. Next, all of the dietary and supplemental intake variables were analysed simultaneously as independent variables in model 2 , including all variables from the base model. We investigated interaction by including interaction terms of dietary and supplemental intakes and smoking status and other covariates. We determined that interaction terms would be significant if the level of significance was $P<0 \cdot 1$. All reported statistical tests were two-sided. Statistical significance was determined using the conventional level of $P<0 \cdot 05$. Statistical analyses were performed using the SPSS statistical software package version $16 \cdot 0$. 


\section{Results}

The mean age of the participants was 66 years (Table 1 ). A large majority reported consuming at least one serving of fruits or vegetables daily, but about half consumed milk products daily or fish more than three times weekly. Reported daily intake of supplements was much less frequent, about $18 \%$ for vitamins A, C, E and D, 6.5\% for $n-3$ PUFA and $2 \cdot 2 \%$ for Se, with almost all of the

Table 1 Characteristics of the study participants: Chinese older adults ( $n$ 2478) aged 55 years and above, Singapore Longitudinal Ageing Studies, 2003-2004

\begin{tabular}{|c|c|c|}
\hline & $n$ or mean & $\%$ or SD \\
\hline Whole sample & 2478 & 100 \\
\hline \multicolumn{3}{|l|}{ Age (years) } \\
\hline Mean and SD & $65 \cdot 9$ & $7 \cdot 6$ \\
\hline \multicolumn{3}{|l|}{ Height $(\mathrm{cm})$} \\
\hline \multicolumn{2}{|l|}{ BMI $\left(\mathrm{kg} / \mathrm{m}^{2}\right)$} & 20 \\
\hline Mean and SD & $23 \cdot 6$ & 3.57 \\
\hline \multicolumn{3}{|l|}{ Gender } \\
\hline Male & 910 & $36 \cdot 9$ \\
\hline Female & 1561 & $63 \cdot 1$ \\
\hline \multicolumn{3}{|l|}{ Housing status } \\
\hline 1-3 room public housing & 709 & $28 \cdot 7$ \\
\hline 4-5 room public housing & 1039 & $41 \cdot 9$ \\
\hline Higher-end public or private & 723 & $29 \cdot 3$ \\
\hline \multicolumn{3}{|l|}{ Smoking } \\
\hline Non-smoker & 2069 & $83 \cdot 5$ \\
\hline Ex-smoker & 252 & $10 \cdot 2$ \\
\hline Current smoker, $<20$ cigarettes daily & 128 & $5 \cdot 1$ \\
\hline Current smoker, $\geq 20$ cigarettes daily & 29 & $1 \cdot 2$ \\
\hline \multicolumn{3}{|l|}{ Reported past or current asthma } \\
\hline Yes & 76 & $3 \cdot 1$ \\
\hline \multicolumn{3}{|l|}{ Past occupational exposure } \\
\hline Yes & 122 & $4 \cdot 9$ \\
\hline \multicolumn{3}{|l|}{ Fruits or vegetables consumption } \\
\hline At least one serving daily & 2274 & $91 \cdot 8$ \\
\hline \multicolumn{3}{|l|}{ Vitamin A supplement } \\
\hline Daily & 113 & $4 \cdot 6$ \\
\hline \multicolumn{3}{|l|}{ Vitamin C supplement } \\
\hline Daily & 286 & $11 \cdot 5$ \\
\hline \multicolumn{3}{|l|}{ Vitamin E supplement } \\
\hline Daily & 207 & $8 \cdot 4$ \\
\hline \multicolumn{3}{|l|}{ Antioxidant vitamins $\mathrm{A} / \mathrm{C} / \mathrm{E}$ supplements } \\
\hline Daily & 462 & $18 \cdot 6$ \\
\hline \multicolumn{3}{|l|}{ Milk or dairy products consumption } \\
\hline At least one serving daily & 1254 & $50 \cdot 6$ \\
\hline \multicolumn{3}{|l|}{ Vitamin D supplement } \\
\hline Daily & 443 & $17 \cdot 9$ \\
\hline \multicolumn{3}{|l|}{ Fish consumption } \\
\hline $\begin{array}{l}\text { More than thrice weekly to daily } \\
n-3 \text { PUFA supplement }\end{array}$ & 1237 & $49 \cdot 9$ \\
\hline Daily & 160 & $6 \cdot 5$ \\
\hline \multicolumn{3}{|l|}{ Se supplement } \\
\hline Daily & 54 & $2 \cdot 2$ \\
\hline \multicolumn{3}{|l|}{ Physical activity score (1-4) } \\
\hline Mean and SD & $2 \cdot 88$ & $0 \cdot 85$ \\
\hline \multicolumn{3}{|l|}{$\mathrm{FEV}_{1}$ (litres) } \\
\hline $\begin{array}{l}\text { Mean and SD } \\
\text { FVC (litres) }\end{array}$ & $1 \cdot 82$ & 0.54 \\
\hline \multicolumn{3}{|l|}{ FVC (litres) } \\
\hline \multicolumn{3}{|l|}{$\mathrm{FEV}_{1} / \mathrm{FVC}(\%)$} \\
\hline Mean and SD & $75 \cdot 4$ & $11 \cdot 5$ \\
\hline
\end{tabular}

$\mathrm{FEV}_{1}$, forced expiratory volume in the first second; FVC, forced vital capacity. remaining proportions reporting no consumption at all. As shown in Table 2, the dietary intakes of fruits/ vegetables, milk/dairy products and fish and supplemental intakes of vitamins A, C, E and D, Se and n-3 PUFA showed a few weak correlations with each other.

Table 3 shows in the base model the expected significant independent associations of gender, age, housing status, smoking, occupational exposure, asthma/COPD history, BMI and physical activity level with $\mathrm{FEV}_{1}, \mathrm{FVC}$ and $\mathrm{FEV}_{1} / \mathrm{FVC}\left(R^{2}=0 \cdot 42\right)$. When added to the base model, supplemental vitamin A, C and $\mathrm{E}$ intakes individually were not significantly associated with $\mathrm{FEV}_{1}$ (data not shown), but together, vitamin A, C and/or E, as well as dietary fish intake and supplemental n-3 PUFA intake showed positive associations with $\mathrm{FEV}_{1}$ (model 1). When all of the dietary and supplemental intakes were analysed simultaneously (model 2), each of them, vitamin A, C and/ or $\mathrm{E}(b=0 \cdot 044, \mathrm{sE}=0 \cdot 022, P=0 \cdot 04)$, dietary fish intake $(b=0 \cdot 058$, SE $0 \cdot 016, P<0 \cdot 0001)$ and supplemental $n-3$ PUFA intake $(b=0 \cdot 068$, sE $=0 \cdot 032, P=0 \cdot 034)$, remained independently associated with $\mathrm{FEV}_{1}$. No significant interactions of dietary or supplemental intakes with smoking or with gender were found.

n-3 PUFA intake showed a significant independent association with FVC $(b=0 \cdot 091$, SE $=0.045, P=0 \cdot 045)$ as well. The results showing the significant associations of individual dietary and supplemental intakes with $\mathrm{FEV}_{1} / \mathrm{FVC}$ (\%) corresponded closely with those for $\mathrm{FEV}_{1}$, except for supplemental $n$-3 PUFA intake, given its positive relationships with both $\mathrm{FEV}_{1}$ and FVC. No significant independent association of fruit/vegetable and milk/dairy product intake and supplemental vitamin D intake with pulmonary function was found.

\section{Discussion}

Evidence in support of the association between consumption of fresh fruits and vegetables and better lung function $^{(7-10)}$ is highly suggestive of the roles of antioxidant vitamins $\mathrm{A}, \mathrm{C}$ and $\mathrm{E}$ in pulmonary protection, but has been found to be inconsistent for different antioxidants across different studies. In the present study, we failed to observe a significant association of dietary intake of vegetables or fruits with pulmonary function. It is known that food sources and the absorption and metabolism of micronutrients in the diet tend to vary in different populations. Among older adults, co-morbid chronic diseases and multiple drug use are common causes of diminished food intake and increased nutritional risks. Infection and chronic diseases perpetuating chronic inflammation and drug use down-regulating nutrient metabolism adversely influence biologically effective concentrations of micronutrients. Hence, although a large majority of participants uniformly reported consuming at least one serving of fruits or vegetables daily, the tissue 
Table 2 Spearman's correlation coefficients among dietary and supplemental intakes of antioxidant and anti-inflammatory nutrients: Chinese older adults ( $n$ 2478) aged 55 years and above, Singapore Longitudinal Ageing Studies, 2003-2004

\begin{tabular}{|c|c|c|c|c|c|c|c|}
\hline & Fruits/vegetables & Milk/dairy products & Fish & Vitamins $\mathrm{A} / \mathrm{C} / \mathrm{E}$ & Vitamin D & Se & $n$-3 PUFA \\
\hline Fruits/vegetables & 1.000 & & & & & & \\
\hline Milk/dairy products & $0 \cdot 133^{\star \star}$ & $1 \cdot 000$ & & & & & \\
\hline Fish & $0.096^{\star \star}$ & 0.039 & $1 \cdot 000$ & & & & \\
\hline Vitamin $\mathrm{A} / \mathrm{C} / \mathrm{E}$ & 0.001 & 0.023 & 0.034 & $1 \cdot 000$ & & & \\
\hline Vitamin D & 0.032 & 0.021 & $0.042^{*}$ & $0.228^{\star \star}$ & 1.000 & & \\
\hline $\mathrm{Se}$ & -0.036 & -0.018 & -0.011 & $0 \cdot 298^{\star \star}$ & $0.226^{\star *}$ & $1 \cdot 000$ & \\
\hline$n-3$ PUFA & 0.013 & 0.003 & 0.001 & $0 \cdot 161^{\star \star}$ & $0.096^{\star \star}$ & $0 \cdot 163^{\star \star}$ & 1.000 \\
\hline
\end{tabular}

${ }^{\star} P<0.05,{ }^{* *} P<0.01$.

Table 3 Multiple regression analysis of relationships of dietary and supplemental micronutrient consumption with $\mathrm{FEV}_{1}, \mathrm{FVC}_{\text {and }} \mathrm{FEV}_{1} /$ FVC: Chinese older adults ( $n$ 2478) aged 55 years and above, Singapore Longitudinal Ageing Studies, 2003-2004

\begin{tabular}{|c|c|c|c|c|c|c|c|c|c|c|c|c|}
\hline & \multicolumn{4}{|c|}{$\mathrm{FEV}_{1}$ (litres) } & \multicolumn{4}{|c|}{ FVC (litres) } & \multicolumn{4}{|c|}{$\mathrm{FEV}_{1} / \mathrm{FVC}(\%)$} \\
\hline & $b$ & SE & $t$ & $P$ & $b$ & SE & $t$ & $P$ & $b$ & SE & $t$ & $P$ \\
\hline \multicolumn{13}{|l|}{ Base model } \\
\hline Intercept & 0.257 & $0 \cdot 229$ & $1 \cdot 119$ & $0 \cdot 26$ & $-0 \cdot 132$ & $0 \cdot 323$ & -0.409 & 0.68 & 0.917 & 0.067 & $130 \cdot 758$ & $<0.0001$ \\
\hline Male gendert & 0.376 & 0.023 & $16 \cdot 553$ & $<0.0001$ & 0.442 & 0.032 & $13 \cdot 813$ & $<0.0001$ & $0 \cdot 016$ & 0.007 & $2 \cdot 408$ & 0.016 \\
\hline Age, single year & -0.025 & 0.001 & $-23 \cdot 592$ & $<0.0001$ & -0.027 & 0.002 & $-18 \cdot 058$ & $<0.0001$ & -0.002 & 0.000 & $-6 \cdot 379$ & $<0.0001$ \\
\hline Height $(\mathrm{cm}) \dagger$ & $2 \cdot 012$ & $0 \cdot 123$ & $16 \cdot 394$ & $<0.0001$ & $2 \cdot 730$ & $0 \cdot 173$ & $15 \cdot 777$ & $<0.0001$ & -0.015 & 0.036 & -0.414 & 0.67 \\
\hline $\operatorname{BMI}\left(\mathrm{kg} / \mathrm{m}^{2}\right)$ & -0.001 & 0.002 & -0.259 & 0.79 & -0.005 & 0.003 & $-1 \cdot 473$ & 0.14 & 0.001 & 0.001 & $1 \cdot 153$ & 0.24 \\
\hline Low-end public housingt & -0.081 & 0.021 & $-3 \cdot 913$ & $<0.0001$ & -0.001 & 0.029 & -0.028 & 0.97 & -0.032 & 0.006 & $-5 \cdot 284$ & $<0.0001$ \\
\hline Mid-range public housingt & -0.052 & 0.019 & $-2 \cdot 766$ & 0.006 & -0.005 & 0.026 & -0.207 & 0.83 & -0.020 & 0.005 & $-3 \cdot 694$ & $<0.0001$ \\
\hline Current smokert & $-0 \cdot 142$ & 0.034 & $-4 \cdot 208$ & $<0.0001$ & 0.010 & 0.047 & $0 \cdot 210$ & 0.83 & -0.056 & 0.010 & $-5 \cdot 729$ & $<0.0001$ \\
\hline Past smokert & -0.046 & 0.028 & $-1 \cdot 640$ & $0 \cdot 101$ & -0.027 & 0.039 & -0.690 & $0 \cdot 49$ & -0.009 & 0.008 & $-1 \cdot 059$ & 0.29 \\
\hline Past occupational exposuret & -0.021 & 0.036 & -0.565 & 0.57 & 0.040 & 0.051 & 0.782 & 0.43 & -0.022 & 0.011 & $-2 \cdot 115$ & 0.035 \\
\hline Reported past or current asthma & -0.341 & 0.045 & $-7 \cdot 539$ & $<0.0001$ & $-0 \cdot 218$ & 0.064 & $-3 \cdot 429$ & 0.001 & -0.084 & 0.013 & $-6 \cdot 428$ & $<0.0001$ \\
\hline Physical activity level $4 \dagger$ & 0.111 & 0.040 & $2 \cdot 758$ & 0.006 & $0 \cdot 145$ & 0.057 & $2 \cdot 552$ & 0.011 & -0.006 & 0.012 & -0.532 & 0.59 \\
\hline Physical activity level 3† & 0.008 & $0 \cdot 021$ & 0.403 & $0 \cdot 68$ & 0.012 & $0 \cdot 030$ & 0.402 & $0 \cdot 68$ & -0.003 & 0.006 & -0.563 & 0.57 \\
\hline Physical activity level $2+$ & -0.025 & 0.020 & $-1 \cdot 296$ & $0 \cdot 19$ & -0.005 & 0.028 & $-0 \cdot 171$ & $0 \cdot 86$ & -0.010 & 0.006 & $-1 \cdot 839$ & 0.066 \\
\hline \multicolumn{13}{|l|}{ Model 1} \\
\hline Fruits or vegetables daily & 0.023 & $0 \cdot 028$ & $0 \cdot 823$ & $0 \cdot 41$ & $0 \cdot 024$ & $0 \cdot 040$ & 0.604 & 0.54 & 0.000 & 0.008 & -0.037 & 0.97 \\
\hline Vitamin A, C or E supplement daily & 0.043 & $0 \cdot 020$ & $2 \cdot 118$ & 0.034 & 0.008 & 0.029 & $0 \cdot 292$ & $0 \cdot 77$ & 0.015 & 0.006 & $2 \cdot 514$ & $0 \cdot 012$ \\
\hline Milk or dairy products daily & -0.019 & 0.016 & $-1 \cdot 215$ & $0 \cdot 22$ & -0.022 & 0.022 & $-1 \cdot 010$ & $0 \cdot 31$ & -0.004 & 0.005 & -0.889 & $0 \cdot 37$ \\
\hline Vitamin D supplement daily & 0.009 & 0.021 & 0.425 & 0.67 & -0.008 & 0.029 & -0.263 & $0 \cdot 79$ & 0.005 & 0.006 & 0.764 & 0.44 \\
\hline Se supplement daily & -0.019 & 0.053 & -0.365 & 0.71 & -0.082 & 0.075 & -1.095 & $0 \cdot 27$ & 0.020 & 0.015 & $10 \cdot 264$ & 0.206 \\
\hline Fish every other day or daily & 0.058 & 0.016 & $3 \cdot 754$ & $<0.0001$ & 0.019 & 0.022 & 0.844 & 0.39 & 0.019 & 0.005 & $4 \cdot 255$ & $<0.0001$ \\
\hline n-3 PUFA supplement daily & 0.070 & 0.032 & $2 \cdot 204$ & 0.028 & $0 \cdot 082$ & 0.045 & $1 \cdot 847$ & 0.065 & 0.006 & 0.009 & 0.683 & 0.49 \\
\hline \multicolumn{13}{|l|}{ Model 2} \\
\hline Fruits or vegetables daily & $0 \cdot 017$ & 0.029 & 0.604 & 0.55 & 0.024 & 0.041 & 0.588 & 0.55 & -0.002 & 0.008 & -0.263 & 0.79 \\
\hline Vitamin $\mathrm{A}, \mathrm{C}$ or $\mathrm{E}$ supplement daily & 0.044 & 0.022 & $2 \cdot 050$ & 0.040 & 0.014 & 0.030 & 0.472 & 0.63 & 0.013 & 0.006 & $2 \cdot 126$ & 0.034 \\
\hline Milk or dairy products daily & -0.023 & 0.016 & $-1 \cdot 484$ & $0 \cdot 138$ & -0.026 & 0.022 & $-1 \cdot 152$ & $0 \cdot 25$ & -0.005 & 0.005 & $-1 \cdot 028$ & $0 \cdot 30$ \\
\hline Vitamin D supplement daily & -0.004 & 0.021 & $-0 \cdot 165$ & $0 \cdot 87$ & -0.008 & 0.030 & -0.277 & $0 \cdot 78$ & -0.001 & 0.006 & $-0 \cdot 102$ & $0 \cdot 91$ \\
\hline Se supplement daily & -0.065 & 0.057 & $-1 \cdot 145$ & 0.25 & $-0 \cdot 111$ & 0.080 & $-1 \cdot 382$ & $0 \cdot 167$ & 0.010 & 0.016 & 0.600 & 0.54 \\
\hline Fish every other day or daily & 0.058 & $0 \cdot 016$ & $3 \cdot 714$ & $<0.0001$ & 0.018 & 0.022 & 0.828 & 0.41 & 0.019 & 0.005 & $4 \cdot 282$ & $<0.0001$ \\
\hline n-3 PUFA supplement daily & 0.068 & 0.032 & $2 \cdot 116$ & 0.034 & 0.091 & 0.045 & $2 \cdot 004$ & 0.045 & 0.003 & 0.009 & 0.331 & 0.74 \\
\hline
\end{tabular}

$\mathrm{FEV}_{1}$, forced expiratory volume in the first second; FVC, forced vital capacity.

Model 1: adjusted for base model variables.

Model 2: adjusted for base model variables and other nutrient variables.

tReferenced to: female gender, higher-end public or private housing, never smoker, no occupational exposure and lowest level (1) of physical activity.

concentration of micronutrients may not be as well correlated with measures of dietary intake as it is with measures of supplement intake. Notably, many studies ignored significant sources of antioxidants and vitamins from supplements in urban populations. In our study population, we measured supplemental intake of antioxidant vitamins and found wide individual variations of intake and little or no correlation with fruit or vegetable intake. We did not detect the effects of vitamin A, C and E supplements when they were analysed individually, but instead observed an effect of these antioxidant vitamins as a group. This appears to be consistent with the orchestrated effect of an array of antioxidants postulated for the known actions of endogenous (and exogenous) antioxidants in the biological defence system against the effects of oxidative stress.

Various studies have reported a positive association ${ }^{(19-22)}$ or no association ${ }^{(4,5)}$ between fish consumption and pulmonary function. No studies as yet have considered simultaneously the effects of $n-3$ PUFA supplements on pulmonary function. We showed that both dietary intake of fish and supplemental intake of $n$-3 PUFA were associated positively with pulmonary function, additionally and independently of each other and other antioxidants. Supplemental $n$-3 PUFA intake was also found to be associated with FVC. This is interesting as it suggests that, perhaps unique among other 
antioxidants, $n-3$ PUFA may both protect against chronic airway narrowing as well as maintain interstitial lung structure and functioning. This should be further studied.

Our findings appeared robust and suggested that the effects associated with these individual antioxidant or antiinflammatory nutrient intakes are non-trivial, considering that the decrement in $\mathrm{FEV}_{1}$ associated with current smoking on average ten cigarettes daily is estimated at $147 \mathrm{ml}$, fish consumption at least thrice weekly with a $57 \mathrm{ml}$ increment in $\mathrm{FEV}_{1}$ and daily $n$-3 PUFA supplement use with a $67 \mathrm{ml}$ increment in $\mathrm{FEV}_{1}$.

We failed to observe results that support the possible antioxidant effect of Se supplements, although the very small number of participants who reported intake of Se should be noted. Another negative result for vitamin D supplement intake is in agreement with a recent study ${ }^{(19)}$ that did not replicate a positive association between vitamin $\mathrm{D}$ and adult pulmonary function reported in a prior study ${ }^{(18)}$.

The limitations in this study should be noted. The use of a semi-quantitative FFQ to assess intake of dietary and supplementary sources of antioxidants and micronutrients is limited, and did not include total energy intake. For certain micronutrients such as Se or vitamin E, dietary intakes are very difficult to measure and unreliable. Milk intake and supplemental vitamin D intake may not be satisfactory surrogate measures of vitamin D status considering that fish is also a rich source of vitamin D, UV exposure from the sun may be highly variable and the elderly are less able to synthesize biologically active 1,25-dihydroxyvitamin D. We did not collect data on the amount of time spent outdoors; hence this limits our statistical analysis for vitamin D. None the less, we were able to observe statistically significant results with the use of self-reports for some dietary and supplemental intakes such as n-3 PUFA. Furthermore, we did not collect information on dosages of supplements used, which could otherwise be useful for comparison against daily nutrient requirements. Further studies involving laboratory measures of biological concentrations of the micronutrients are desirable. Multiple testing involving many nutritional variables may give rise to spurious statistical significance; however, observed $P$ values $<0.0001$ are not likely to be type one errors.

With cross-sectional results, the observed associations may arguably be confounded by dietary change resulting from poor pulmonary function. In patients with COPD, this is generally expected to result in reduced food intake and undernutrition. Community-living older persons possessing varying levels of pulmonary function include a sub-population of individuals who have COPD and poor lung function, but it is well known that it is generally unrecognizable and it seems very unlikely that these individuals would accordingly increase their dietary intake.

The strengths of the present study include its large sample size and the selection of an older adult population who are vulnerable to the effects of oxidative injury and nutrient deficiency and are at elevated risk of COPD. We controlled for a large number of known risk factors for COPD that were potentially confounding variables in multivariate analyses and observed robust relationships with pulmonary function that were expected. We measured dietary and supplementary intakes of multiple antioxidants and anti-inflammatory nutrients, because a protective effect attributed to one antioxidant or micronutrient may actually reflect the effect of another correlated dietary constituent or an interaction between dietary constituents.

This Asian population-based study lends further support to the hypothesis that antioxidant and anti-inflammatory micronutrients have protective effects on pulmonary function. The results suggest that fish consumption and supplemental intakes of $n-3$ PUFA and vitamins A, C and E likely have beneficial effects on respiratory health and protection against the risk of COPD in older persons.

\section{Acknowledgements}

Sources of funding: The Singapore Longitudinal Ageing Study was supported by grants (no. 03/1/21/17/214 and no. 08/1/21/19/567) from the Biomedical Research Council of the Agency for Science, Technology and Research (A*STAR) in Singapore. A*STAR had no role in the design, analysis or writing of this article. Conflict of interest: None of the authors report conflicts of interest with commercial enterprises. Authors' contributions: T.P.N. conceptualized and designed the study, formulated the hypothesis, performed the statistical analysis and results, and drafted and revised the manuscript. M.N. participated in the study design and the statistical analysis, interpretation of the results and review of the manuscript. K.B.Y. and W.C.T. participated in the study design, interpretation of the results and review of the manuscript. T.P.N. is the guarantor of the paper, taking responsibility for the integrity of the work as a whole, from inception to published article. Acknowledgements: The authors thank the participants in South East Singapore, Geylang East Home for the Aged, Presbysterian Community Services, Thye Hua Kuan Moral Society, Henderson Senior Citizens' Home, and People's Association for their support and assistance in the study.

\section{References}

1. Romieu I \& Trenga C (2001) Diet and obstructive lung diseases. Epidemiol Rev 23, 268-287.

2. Schünemann HJ, Freudenheim JL \& Grant BJB (2001) Epidemiologic evidence linking antioxidant vitamins to pulmonary function and airway obstruction. Epidemiol Rev 23, 248-267.

3. Strachan D, Cox B, Erzinclioglu S et al. (1991) Ventilatory function and winter fresh fruit consumption in a random sample of British adults. Thorax 46, 624-629.

4. Butland B, Fehily A \& Elwood P (2000) Diet, lung function, and lung function decline in a cohort of 2512 middle aged men. Thorax 55, 102-108. 
5. Tabak C, Smit H, Rasanen L et al. (1999) Dietary factors and pulmonary function: a cross sectional study in middle aged men from three European countries. Thorax 54, 1021-1026.

6. Carey I, Strachan D \& Cook D (1998) Effects of changes in fresh fruit consumption on ventilatory function in healthy British adults. Am J Respir Crit Care Med 158, 728-733.

7. Grievink L, Smit HA, Ocke MC et al. (1998) Dietary intake of antioxidant (pro)-vitamins, respiratory symptoms and pulmonary function: the Morgen study. Thorax 53, 166-171.

8. Britton JR, Pavord ID, Richards KA et al. (1995) Dietary antioxidant intake and lung function in the general population. Am J Respir Crit Care Med 151, 1383-1387.

9. Hu G \& Cassano PA (2000) Antioxidant nutrients and pulmonary function: the Third National Health and Nutrition Examination Survey (NHANES III). Am J Epidemiol 151, 975-981.

10. Bentley AR, Kritchevsky SB, Harris TB et al. (2012) Dietary antioxidants and forced expiratory volume in $1 \mathrm{~s}$ decline: the Health, Aging and Body Composition study. Eur Respir J 39, 979-984.

11. McKeever TM, Lewis SA, Smit HA et al. (2008) A multivariate analysis of serum nutrient levels and lung function. Respir Res 29, 67.

12. Schunemann HJ, Grant BJ, Freudenheim JL et al. (2001) The relation of serum levels of antioxidant vitamins $C$ and $\mathrm{E}$, retinol and carotenoids with pulmonary function in the general population. Am J Respir Crit Care Med 163, 1246-1255.

13. Grievink L, de Waart FG, Schouten EG et al. (2000) Serum carotenoids, $\alpha$-tocopherol, and lung function among Dutch elderly. Am J Respir Crit Care Med 161, 790-795.
14. Guenegou A, Leynaert B, Pin I et al. (2006) Serum carotenoids, vitamins $\mathrm{A}$ and $\mathrm{E}$, and 8 year lung function decline in a general population. Thorax 61, 320-326.

15. Pearson $\mathrm{P}$, Britton J, McKeever $\mathrm{T}$ et al. (2005) Lung function and blood levels of copper, selenium, vitamin C and vitamin E in the general population. Eur J Clin Nutr 59, 1043-1048.

16. Koli K \& Keski-Oja J (2000) 1- $\alpha, 25$-Dihydroxyvitamin $\mathrm{D}_{3}$ and its analogues down-regulate cell invasion-associated proteases in cultured malignant cells. Cell Growth Differ 11, 221-229.

17. Dobak J, Grzybowski J, Liu FT et al. (1994) 1,25Dihydroxyvitamin $\mathrm{D}_{3}$ increases collagen production in dermal fibroblasts. J Dermatol Sci 8, 18-24.

18. Black PN \& Scragg R (2005) Relationship between serum 25-hydroxyvitamin D and pulmonary function in the third national health and nutrition examination survey. Chest 128, 3792-3798.

19. Shaheen SO, Jameson KA, Robinson SM et al. (2011) Relationship of vitamin D status to adult lung function and COPD. Thorax 66, 692-698.

20. Kelly Y, Sacker A \& Marmot M (2003) Nutrition and respiratory health in adults: findings from the health survey for Scotland. Eur Respir J 21, 664-671.

21. Sharp D, Rodriguez B, Shahar E et al. (1994) Fish consumption may limit the damage of smoking on the lung. Am J Respir Crit Care Med 150, 983-987.

22. Shahar E, Folsom AR, Melnick SL et al. (1994) Does dietary vitamin A protect against airway obstruction? The Atherosclerosis Risk in Communities (ARIC) Study Investigators. Am J Respir Crit Care Med 150, 978-982.

23. Schwartz J \& Weiss ST (1994) The relationship of dietary fish intake to level of pulmonary function in the First National Health and Nutrition Examination Survey (NHANES I). Eur Respir J 7, 1821-1824. 